\title{
USO DE FACTURACIÓN ELECTRÓNICA EN APLICACIÓN SAP R/3 BAJO METODOLOGÍA TRADICIONAL (CASCADA)
}

Used of sap $r / 3$ electronic invoicing applications under waterfall traditional methodology

\author{
Iván Ho \\ Universidad de Panamá, Facultad de Administración de Empresas y Contabilidad, Panamá \\ Email: ivanhog@gmail.com (iD https://orcid.org/0000-0001-8634-0863 \\ Iván Clarence \\ Universidad Tecnológica de Panamá, Facultad de Ingeniería de Sistemas, Panamá \\ Email: ivan.clarence@yahoo.com iD https://orcid.org/0000-0003-3288-6443

\section{Yussibel Bedoya} \\ Universidad Tecnológica de Panamá, Facultad de Ingeniería de Sistemas, Panamá \\ Email: yussibel.bedoya@hotmail.com (D) https://orcid.org/0000-0002-1671-2921

\section{Marcos Arjona} \\ Universidad Tecnológica de Panamá, Facultad de Ingeniería de Sistemas, Panamá \\ Email: marcosfa 8@hotmail.com (iD) https://orcid.org/0000-0002-3735-4780
}

\section{RESUMEN}

Las instituciones en Panamá han tenido que enfrentarse a transformaciones radicales a nivel de procesos de digitalización. La facturación electrónica otorga a las compañías la capacidad de automatizar el procesamiento de sus facturas, por lo que todo el ecosistema empresarial se favorece de su implementación. A través de implementaciones de los sistemas informáticos SAP R/3 de facturación electrónica, los certificados digitales, EDI e IDOC. El objetivo principal de la facturación electrónica se fundamenta en agilizar la compra de bienes y servicios, mejorando los procesos de administración y auditoría que las instituciones gubernamentales operan sobre las empresas. La idea principal es que esta investigación sirva de referencia para implementaciones en aplicaciones SAP R/3 en diferentes empresas, sobre todo en organizaciones multinacionales donde por temas de seguridad y cumplimiento, los accesos a la aplicación SAP R/3 son más restringido de forma directa, por lo que la única forma de conectar SAP R/3 con aplicaciones de terceros es a través de interfaces donde se intercambian archivos planos o IDOC.

Palabras claves: certificados digitales, EDI, IDOC, facturación electrónica, SAP R/3.

\begin{abstract}
Institutions in Panama have had to face radical transformations at the level of digitization processes. Electronic invoicing empowers companies to automate the processing of their invoices, so the entire business ecosystem favors their implementation. Through implementations of the SAP R/3 electronic invoicing computer systems, digital certificates, EDI, and IDOC. The main objective of electronic invoicing is based on streamlining the purchase of goods and services, improving the administration and audit processes that government institutions operate on companies. The main idea is that this research serves as a reference for implementations in SAP R/3 applications in different companies, especially in multinational organizations where due to security and compliance issues, access to the SAP $\mathrm{R} / 3$ application is more directly restricted, so the only way to connect SAP R/3 with third-party applications is through interfaces where flat files or IDOC's are exchanged.
\end{abstract}

Keywords: digital certificates, EDI, IDOC, electronic invoicing, SAP R/3 

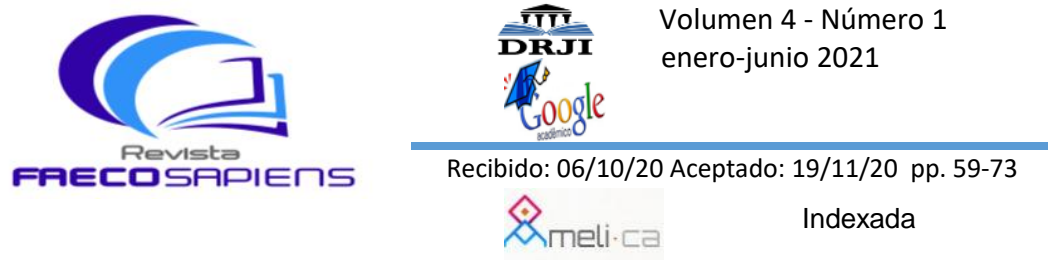

\section{INTRODUCCIÓN}

En un mundo, cada día más globalizado y aprovechando el enorme desarrollo de tecnologías informáticas, las autoridades tributarias de cada país se abocan a la utilización de una factura electrónica, lo que permite no solo tener un mayor control fiscal para la agencia gubernamental, sino también un ahorro en papelería y tiempo para las empresas y los consumidores.

Este trabajo investigativo va enfocado en demostrar que con el uso adecuado de tecnologías y bajo un ambiente de manejo de proyectos tradicional (cascada), que posibilitan la conexión entre las interfaces (entradas y salidas) de SAP R/3 con una aplicación en ambiente web, que sea capaz de poder utilizar los mensajes EDI-SAP (Electronic Data Interchange-Systems, Applications, Products in Data Processing), y convertirlos en facturas digitales que puedan intercambiar con los servidores de la autoridad tributaria.

El tema de la facturación electrónica no es nuevo para Latinoamérica, ya que en las últimas dos décadas cada vez más países han adoptado esta metodología tributaria para el envío y recepción de facturas. El país pionero fue Chile en 2003, y de allí lo han seguido países como Argentina, Brasil, Ecuador, México, Perú, Uruguay, Colombia, Costa Rica, y más recientemente en Rep. Dominicana como lo demuestra el estudio de la Evolución Factura Electrónica en América Latina del 2014. (Figura 1)

\section{Figura 1.}

Evolución de Factura Electrónica en Latinoamérica.

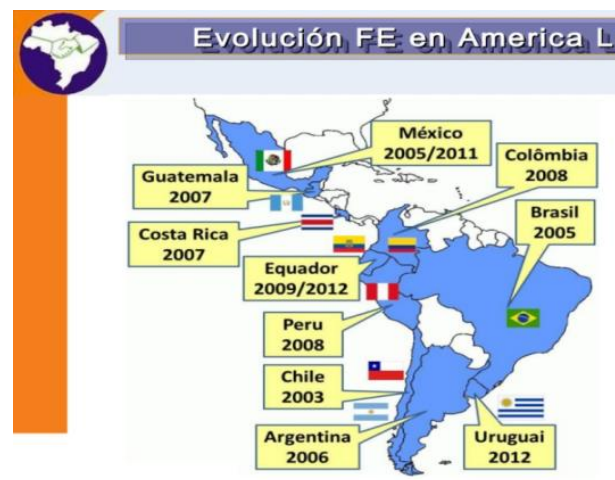

Nota: La figura presenta la evolución de la factura electrónica de América Latina incluyendo los años de implementación. Recuperado de https://dgi.mef.gob.pa/facturaelectronica/index.html, 2019 
REVISTA FAECO SAPIENS

ISSN L 2644-3821

Acceso Abierto. Disponible en:

https://revistas.up.ac.pa/index.php/faeco sapiens
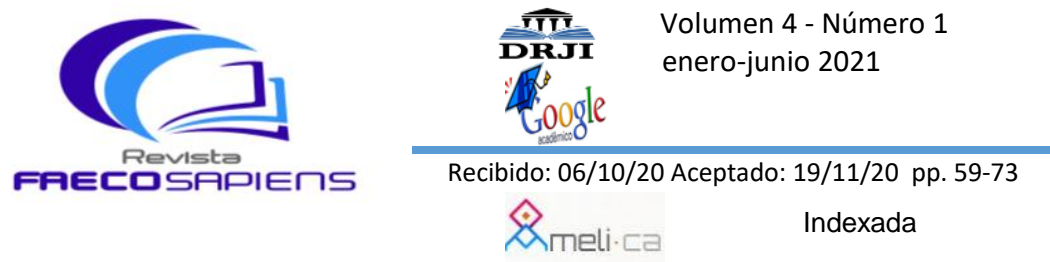

\section{SAP R/3}

El valor de esta investigación radica en conseguir una base para la correcta implementación del sistema de facturación electrónica utilizando el sistema SAP. Tal como lo menciona Angy Mejias en su informe "Interoperabilidad de módulos del sistema R/3 de SAP es: "una corporación en el ámbito mundial. Fundada en 1972 y con sede en Walldorf, Alemania, SAP es la cuarta compañía mundial en ventas de software en el mundo. La compañía SAP fue fundada por cuatro antiguos empleados de IBM y hoy es una empresa multinacional, teniendo presencia en más de 50 países alrededor del mundo".

Este proyecto sirve de referente para determinar los componentes que forman parte de la implementación de la factura electrónica utilizando SAP. Podemos mencionar que "SAP fue uno de los primeros desarrolladores de software en administración empresarial. Debido a esto, los sistemas de administración integral de SAP tales como sus sistemas $\mathrm{R} / 2$ y $\mathrm{R} / 3$, son los más utilizados alrededor del mundo. Ambos sistemas tienen el respaldo de un sólido conocimiento en administración y organización comercial. El sistema R/2 de SAP fue previo a la salida de su sistema $\mathrm{R} / 3$ en 1992. Este sistema principalmente está enfocado en la unidad central de cómputo, mientras que su sistema $\mathrm{R} / 3$ es orientado a la arquitectura cliente/servidor. El sistema R/3 de SAP es uno de los sistemas denominados ERP" como lo puntualiza Mejías, A. (2013) en su obra la Interoperabilidad de módulos del sistema $\mathrm{R} / 3$ de SAP. En Panamá, existen múltiples empresas privadas utilizando el sistema SAP/R3.

\section{ERP}

Podemos mencionar, que dentro de portafolios de productos SAP se destaca el del ERP. "El término ERP se refiere a Enterprise Resource Planning, que significa sistema de planificación de recursos empresariales". Estos programas se hacen cargo de distintas operaciones internas de una empresa, desde producción a distribución o incluso recursos humanos.

Uno de los grandes retos de todas las empresa es realizar de forma exitosa la implementación de un nuevo sistemas informático. Los sistemas ERP suponen una gran inversión para las empresas. Según una encuesta de "Panorama Consulting de 2013, un $40 \%$ de las empresas que adquieren un sistema ERP notan un aumento en la productividad" como lo estable Tic Portal, (2019). Además, los módulos pueden incluir aplicación para las finanzas, contabilidad, tesorería, operaciones, mercadeos, etc. 
REVISTA FAECO SAPIENS

ISSN L 2644-3821

Acceso Abierto. Disponible en:

https://revistas.up.ac.pa/index.php/faeco sapiens
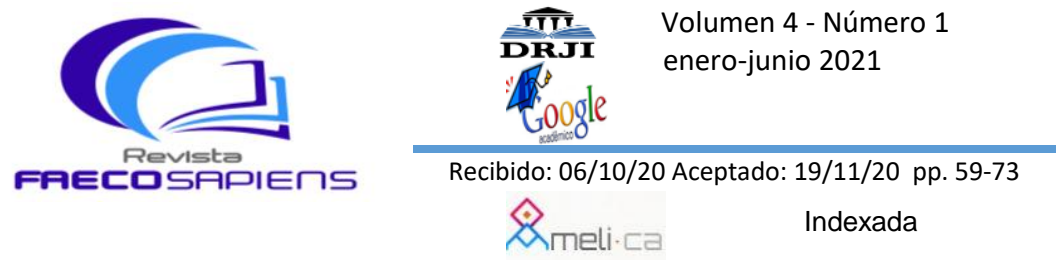

\section{IDOC}

Un IDOC es: un formato de SAP estándar para el intercambio electrónico de datos entre sistemas. "IDOC es simplemente un contenedor de datos utilizado para intercambiar información entre dos procesos que pueden comprender la sintaxis y la semántica de los datos. En palabras simples, un IDOC es como un archivo de datos con un formato específico que se intercambia entre dos sistemas que saben cómo interpretar esos datos. IDOC significa" documento intermedio.

Para ponernos en contexto SAP no trabaja directamente con los lenguajes EDIFACT ni datos estructurados de intercambio electrónicos de datos. "Cuando se ejecuta una saliente (outbound) del proceso intercambio de datos de forma electrónica (EDI), un IDOC es creado. En una entrante (inbound) del proceso EDI, un IDOC sirve como entrada para crear un documento en la aplicación. En el sistema SAP, los IDOCs se almacenan en la base de datos".

EI IDOC permite relacionar la información entre distintos sistemas. Así como los datos de un distribuidor o una oferta "cada IDOC tiene un único número (dentro de un cliente). Los IDOCs se basan en estándares EDI, ANSI ASC X12 y EDIFACT. En caso de cualquier conflicto en el tamaño de los datos, adopta uno con mayor longitud. Los IDOCs son independientes de la dirección del intercambio de datos, por ejemplo, ORDERS01: Módulo de compras: entrantes y salientes. Los IDOCs se pueden ver en un editor de texto. Los datos se almacenan en formato de caracteres en lugar de formato binario. Los IDOCs son independientes de los sistemas de envío y recepción (SAP y No-SAP)", como lo indica el portal Guru99 (2019).

\section{EDI}

Lo que diferencia a los EDI de otras plataformas de intercambio de datos es la homogenización de la información. "El Intercambio Electrónico de Datos, también conocido como EDI, es un sistema de comunicación que permite el envío y la recepción de documentos electrónicos en un formato normalizado entre los sistemas informáticos de quienes intervienen en una relación comercial. El modelo de trabajo EDI implica la realización de acciones comerciales sin papeles, de forma electrónica, mediante una estructura específica y sobre un estándar internacional" como lo explica en su portal del Seres Grupo Dacaposte (2020).

Gracias a los EDI, los documentos se pueden intercambiar de forma electrónica entre los diferentes sistemas gracias a un solo lenguaje de programación común que permite el intercambio de la información entre sí. El programa SAP no trabaja de 


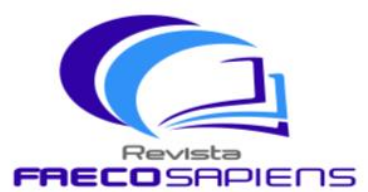

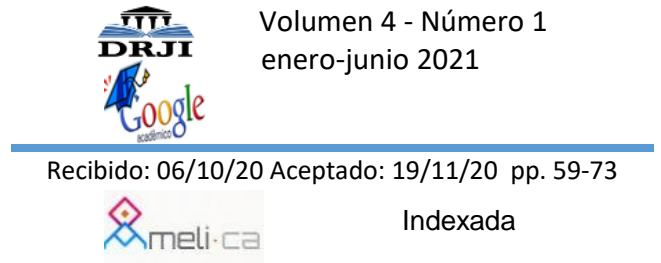

forma directa con el lenguaje de programación EDIFACT. "SAP tiene sus propios mensajes llamados IDOCs. Estos IDOCs son similares a los mensajes EDI, pero tienen sus propias estructuras. Normalmente estos IDOCs de SAP trabajan en XML para el intercambio de datos. Por tanto, SAP ya considera XML como su estándar principal para el intercambio de datos. La tendencia es que el XML sustituya a medio plazo a EDIFACT ya que este último presenta muchas ventajas al permitir identificar cualquier dato, lo que en edifact no es posible".

El que SAP pueda reconocer al formato EDI es posible al ejecutar una conversión. "EDI - SAP IDOC" o "SAP IDOC - EDI. Por ejemplo: Cuando se recibe un pedido EDI ORDERS en SAP se traduce del lenguaje EDIFACT a XML (utilizando un esquema predefinido) y se convierte en un IDOC que se integra en el módulo de pedidos. Cuando se emite un DESADV o INVOIC desde SAP, se genera un IDOC que se convierte a XML y finalmente se traduce a EDIFACT para su envío ya que por el momento este último lenguaje es el más extendido entre proveedores y clientes". La herramienta que utiliza SAP para realizar estas conversiones o transformaciones es SAP Netweaver Process Orchestration (SAP PO).

Figura 2.

SAP PO

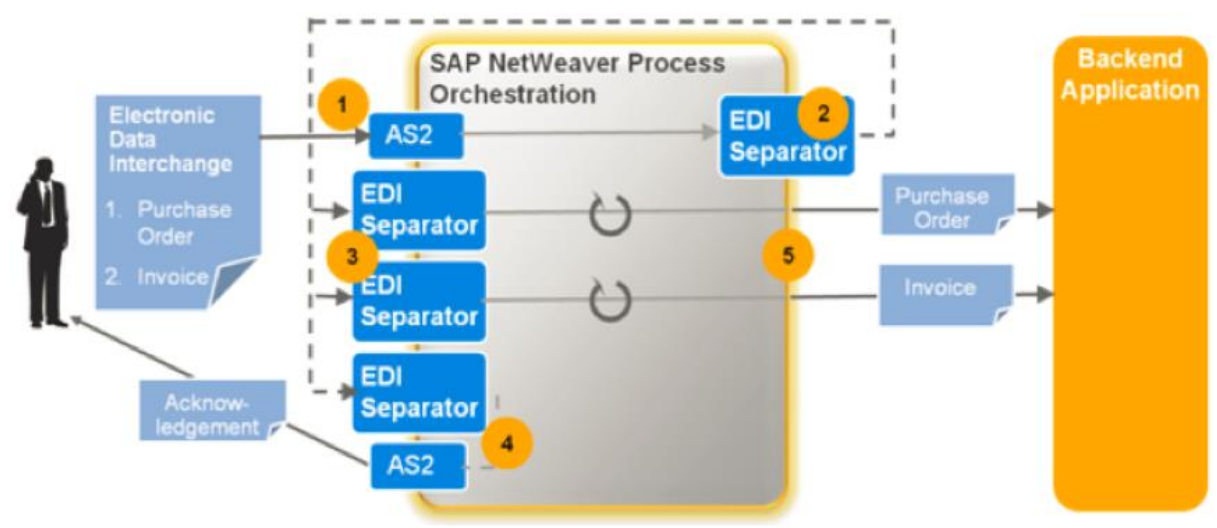

Nota: La influencia del SAP en el intercambio de información. Recuperado de: https://www.sothis.tech/intercambio-informacion-ediintegracion-sap/, 2019.

SAP PO otorga todas las características de los sistemas informáticos que las empresas necesitan al momento de migrar los flujos de procesos empresariales. Una diferente alternativa para realizar la transformación entre los mensajes "EDI y los IDOCs en SAP es hacerlo mediante proveedores externos los cuales manejan la comunicación entre ambos sistemas y la conversión de formatos". 
REVISTA FAECO SAPIENS

ISSN L 2644-3821

Acceso Abierto. Disponible en: https://revistas.up.ac.pa/index.php/faeco sapiens
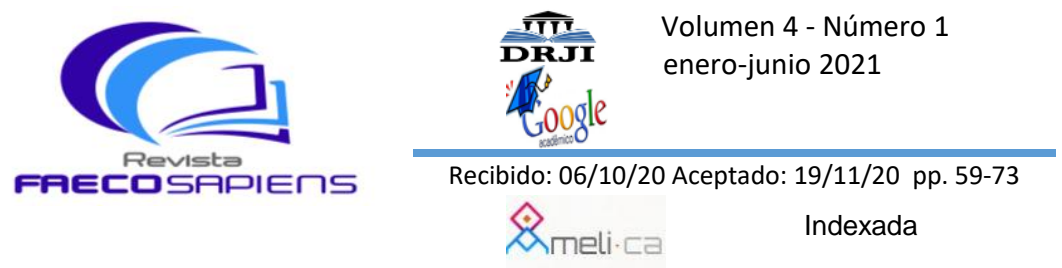

Figura 3.

IDOC EDI.

\section{data}

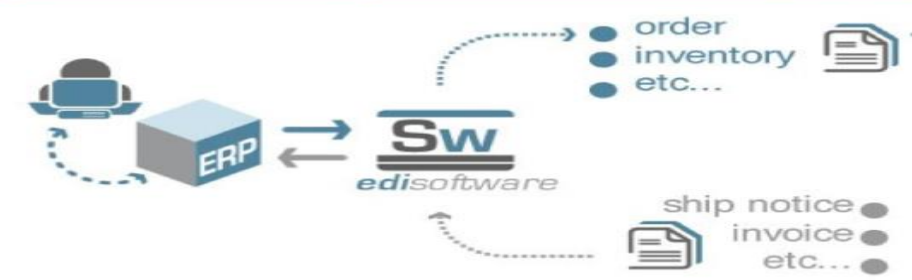

\section{standardization / mapping}

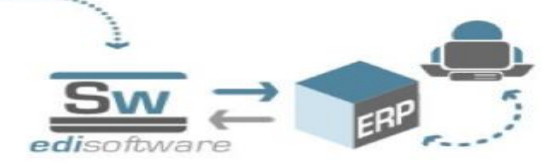

integration

Nota: La integración, estandarización e integración de información. Recuperado de: https://www.sothis.tech/intercambioinformacion-edi-integracion-sap/, 2019

Según Escudero (2017), cuando se ejecuta la integración de los sistemas, "SAP tiene multitud de herramientas para realizar la monitorización, alertas, reproceso de mensajes y conexión. Estas herramientas nos permiten poder ver todo el flujo de los mensajes desde el origen hasta el destino, monitorear por cliente/proveedor, visualizar los documentos creados en SAP (pedidos, entregas, facturas). Una de estas herramientas es la llamada SAP AIF (Application Interface Framework). Esta herramienta permite realizar el seguimiento y monitorización de todas las interfaces en SAP (EDI y no EDI). Además, también permite realizar mapeos y conversiones fácilmente. Se pueden realizar el seguimiento de todas las interfaces por cliente, aplicación de negocio, de una forma muy visual y adaptada al usuario final".

\section{Factura Electrónica}

Según el portal web de Debitoor (2019): la facturación electrónica "es una revolución frente a la factura tradicional en papel y consiste en la transmisión de las facturas o demás comprobantes entre emisor y receptor por medios de comunicación electrónicos. Características de la factura electrónica Estas e-facturas incluyen las características y requisitos de las facturas tradicionales, pero que al ser electrónica, autentifica tanto el emisor como el contenido de la misma y le confiere veracidad".

Para la realización y envío de factura electrónica, ha de poseer firma digital (Certificado Digital). Formatos de la factura electrónica. Los formatos varían considerablemente de país a país en materia de factura electrónica. Estos formatos 
REVISTA FAECO SAPIENS

ISSN L 2644-3821

Acceso Abierto. Disponible en:

https://revistas.up.ac.pa/index.php/faeco sapiens

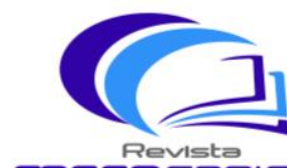

FPECDSAPIERS

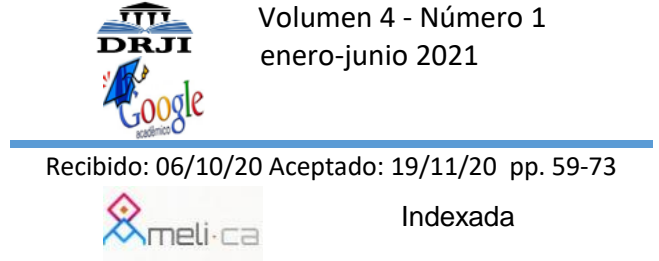

o guías codificadas se extraen a partir del EDI (Electronic Data Interchange). El EDIFACT (Interchange for Administration, Commerce and Transport) Intercambio Electrónico de Datos para la Administración, Comercio y Transporte, "es el estándar EDI internacional desarrollado por las Naciones Unidas. Este estándar establece un conjunto de reglas de sintaxis que se usan para estructurar el protocolo de intercambio interactivo. Permite un intercambio seguro de documentos y archivos comerciales a través de múltiples países e industrias".

El formato EDIFACT obedece a unos lineamientos de estructura de datos y normalización de mensajes para el intercambio de la información. "Se compone de estándares, directorios y lineamientos acordados internacionalmente, diseñados en específico para el intercambio electrónico de datos estructurados. Está diseñado para realizar transferencias de archivos y documentos relacionados al comercio de bienes y servicios, entre sistemas de información computarizados que están separados entre sí" como se señala en el portal goanywhere.com (2019).

El formato EDIFACT, se utiliza cuando se envía la factura de ordenador a ordenador, y el receptor tiene la intención o quiere la posibilidad de modificar la información y tratarla. Por otro lado, cuando el receptor de la factura - un cliente, por ejemplo - sólo quiere guardarla sin modificar los datos, el formato más utilizado es el formato PDF. La factura electrónica, es un tipo de factura que se diferencia de la factura en papel por la forma de gestión informática y el envío mediante un sistema de comunicaciones que conjuntamente permiten garantizar la autenticidad y la integridad del documento electrónico.

Entre las muchas ventajas que ofrece la facturación electrónica, encontramos:

1. "Obtención de información en tiempo real.

2. Reducción de tiempos de gestión; al estar todo informatizado cualquier cambio en la facturación no requiere apenas tiempo.

3. Uso eficaz de los recursos financieros.

4. Integración con aplicaciones internas de empresas.

5. Mayor control de acciones erróneas; con las herramientas de gestión y las certificaciones digitales, el riesgo de error se reduce de manera considerable. Entre ellos el error humano.

6. Menor destrucción del medio ambiente, principalmente con la grandísima reducción en la tala de árboles", como lo describe Muñoz, M. (2014). 
REVISTA FAECO SAPIENS

ISSN L 2644-3821

Acceso Abierto. Disponible en:

https://revistas.up.ac.pa/index.php/faeco sapiens
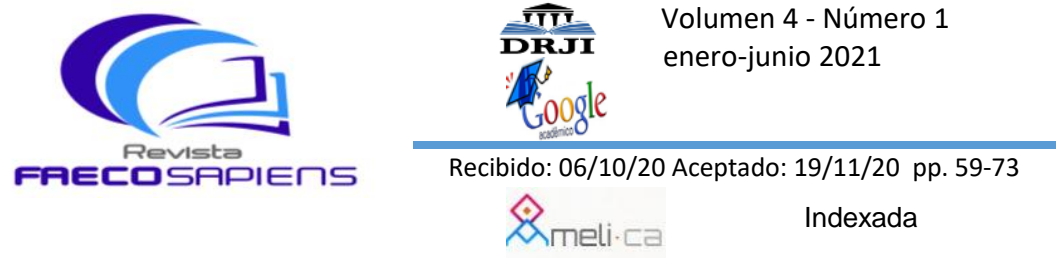

\section{Certificado Digital}

El Certificado Digital permite la identificación exclusiva de una entidad. En esencia permiten a un usuario verificar a quién se ha emitido un certificado, así como el emisor del certificado. "Es el único medio que permite garantizar técnica y legalmente la identidad de una persona en internet. Se trata de un requisito indispensable para que las instituciones puedan ofrecer servicios seguros a través de internet. Además: el certificado digital permite la firma electrónica de documentos. El receptor de un documento firmado puede tener la seguridad de que éste es el original y no ha sido manipulado y el autor de la firma electrónica no podrá negar la autoría de esta firma".

El certificado digital contiene información acerca de la identidad del propietario del certificado y acerca de la autoridad de certificación. "El certificado digital permite cifrar las comunicaciones. Solamente el destinatario de la información podrá acceder al contenido de la misma. En definitiva, la principal ventaja es que disponer de un certificado le ahorrará tiempo y dinero al realizar trámites administrativos en Internet, a cualquier hora y desde cualquier lugar. Un Certificado Digital consta de una pareja de claves criptográficas, una pública y una privada, creadas con un algoritmo matemático, de forma que aquello que se cifra con una de las claves sólo se puede descifrar con su clave pareja" como lo explica en el artículo ¿Qué es un Certificado Digital? de la Universidad Politécnica de Valéncia. (2012).

\section{Figura 4}

\section{Certificado Digital}

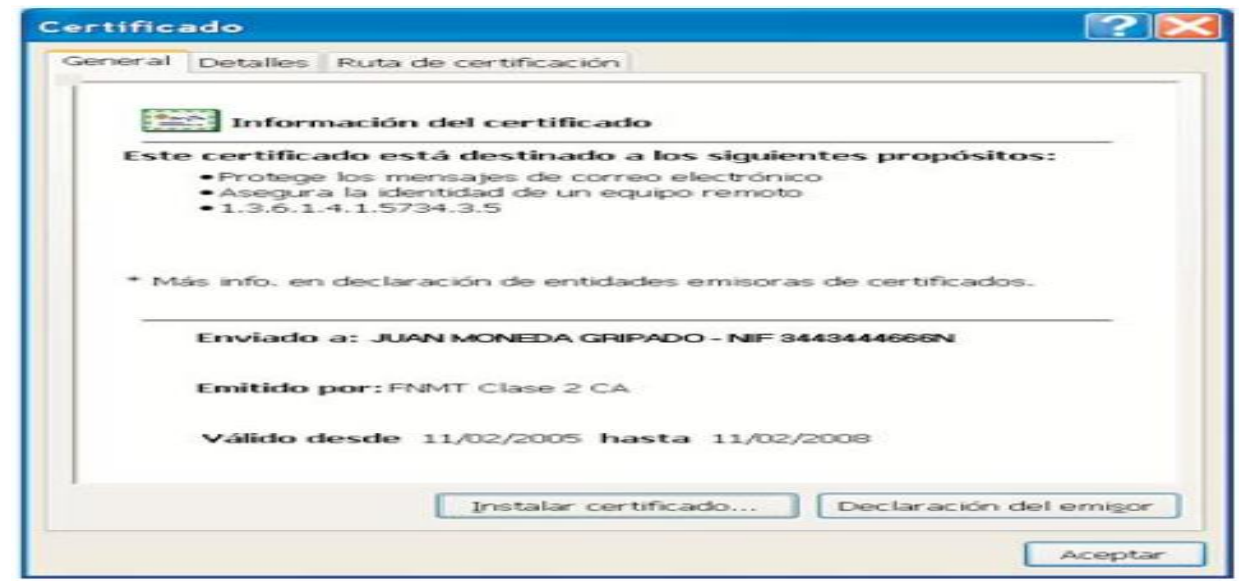

Nota: Es la pantalla que muestra el certificado de protección del correo electrónico. Recuperado de: https://www.upv.es/contenidos/CD/info/711545normalc.html, 2019 
Acceso Abierto. Disponible en:
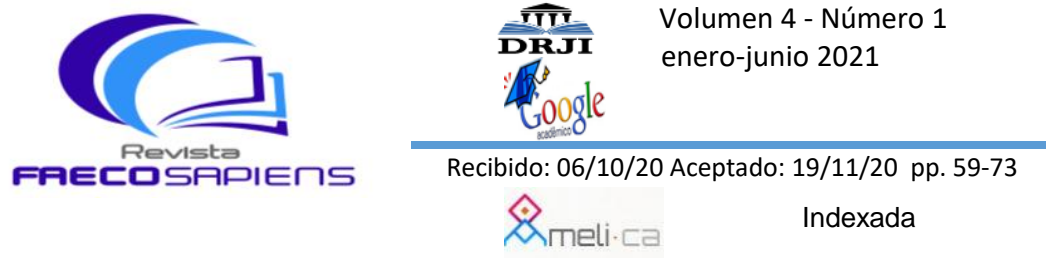

\section{Metodología de implementación}

Esta investigación da a conocer las ventajas y beneficios de la implementación de la factura electrónica utilizando la metodología cascada. "También conocido como modelo clásico, modelo tradicional o modelo lineal secuencial. Él método de la cascada es considerado como el enfoque clásico para el ciclo de vida del desarrollo de sistemas, se puede decir que es un método puro que implica un desarrollo rígido. Está es una secuencia de actividades (o etapas) que consisten en el análisis de requerimientos, él diseño, la implementación, la integración y las pruebas".

- "El análisis de requerimientos consiste en reunir las necesidades del producto y casi siempre su salida es texto.

- El diseño describe la estructura interna del producto y suele representarse con diagramas y texto.

- La implementación significa programación. Producto de esta etapa es el código en cualquier nivel, incluido el producido por sistemas de generación automática.

- La integración es el proceso de ensamblar las partes para completar el producto" como se detalla en el Blog de Solorio (2013).

\section{Figura 5.}

\section{Etapas en la Metodología de Cascada}

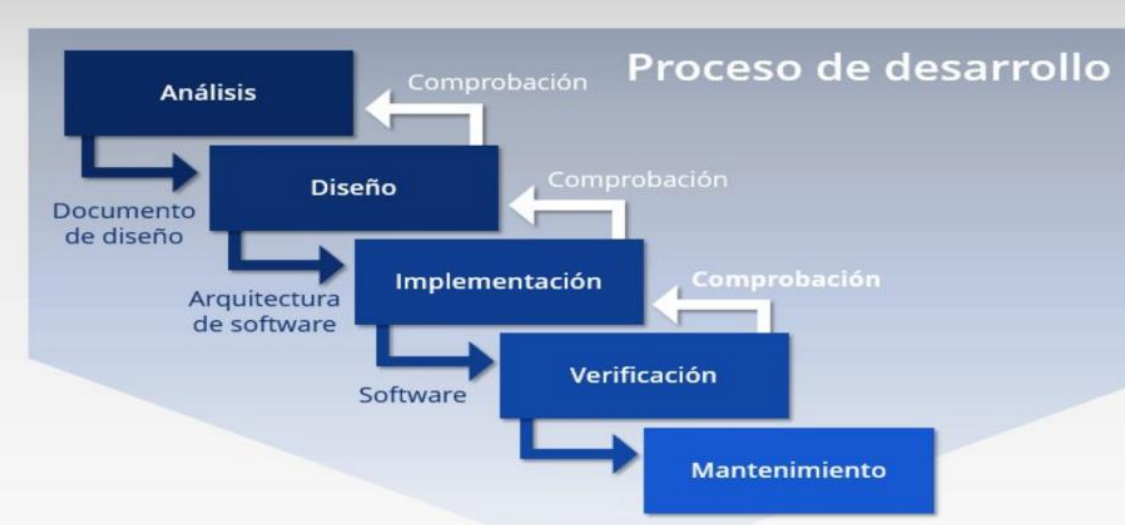

Nota: El modelo en cascada: desarrollo secuencial de software, https://www.ionos.es/digitalguide/paginasweb/desarrollo-web/el-modelo-en-cascada/,

Revisado: 11/03/2019. 

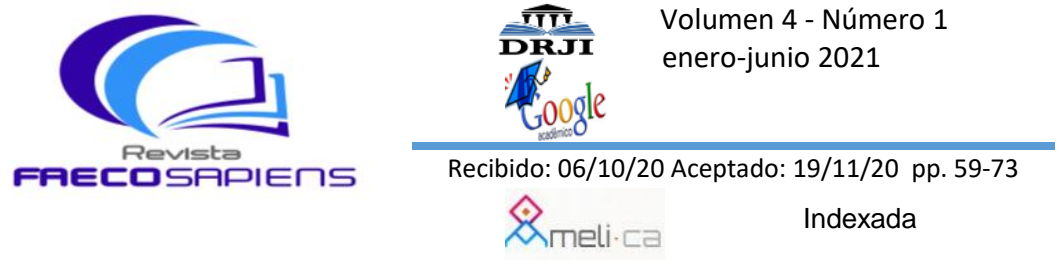

Los procesos de implementación de la factura electrónica en SAP se vienen realizando con la metodología cascada desde hace varios años. "Los requerimientos para la implementación de este sistema se deben definir, se diseña la solución, se implementa, se prueba y se ejecuta el programa en productivo. El proceso completo puede durar semanas o meses, dependiendo de la complejidad del requerimiento. La calidad del proceso generalmente se mide según dos criterios: 1) si la ejecución se ajustó a lo planificado, en términos de esfuerzos y plazos, y 2) si el producto final no presenta fallas" como se describe en el artículo una metodología ágil para las mejoras de SAP del portal de Novis (2018).

\section{Figura 6}

Proceso de Facturación en el Sistema SAP/R3

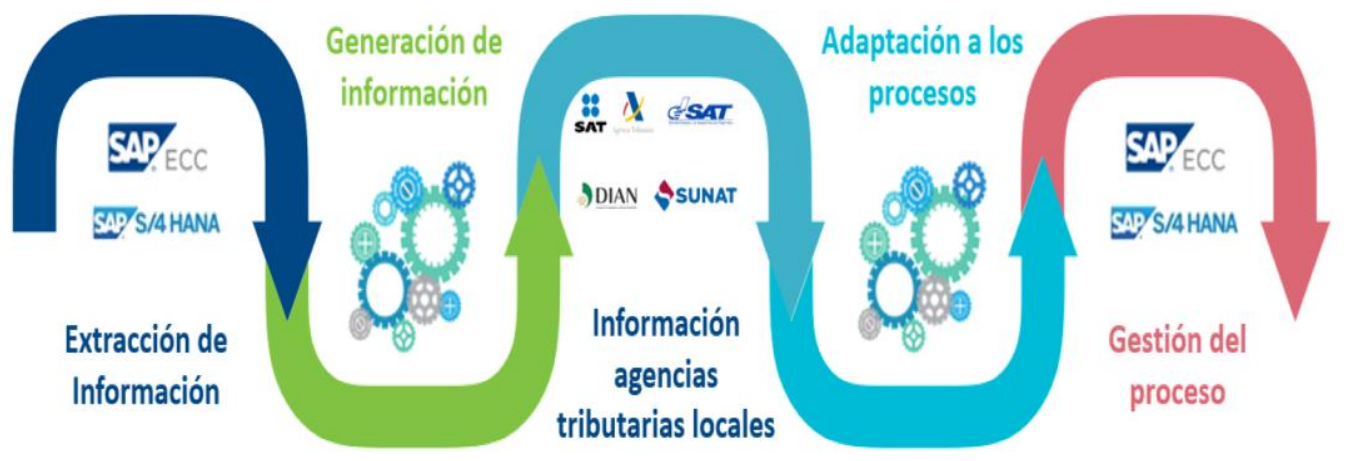

Nota: Rafael Luque, Guía práctica para la implementación de factura electrónica, 2019.

\section{Resultados de la implementación}

La presente investigación permitirá contribuir a realizar un marco de referencia en la implementación de la facturación electrónica utilizando el programa SAP a través de la metodología cascada. Pero antes de iniciar cualquier proyecto se debe contar con un "Caso del Negocio" que contenga la justificación del proyecto y la documentación que sustente su implementación. 

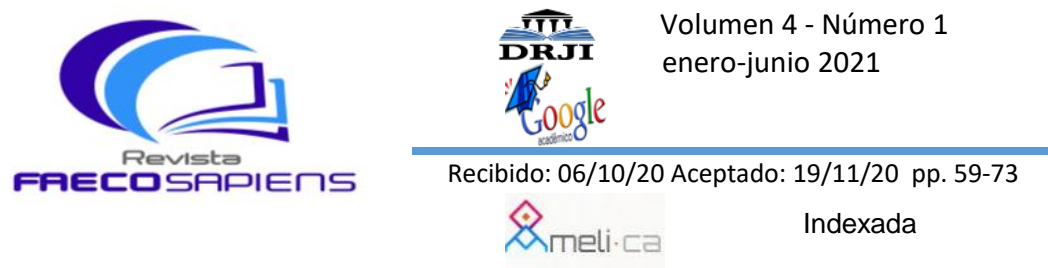

A continuación, detallaremos las actividades a desarrollar en cada una de las etapas del proyecto:

- Análisis.

- Diseño.

- Construcción.

- Pruebas.

- Documentación y entrenamiento.

- Puesta en marcha del sistema y cierre del proyecto.

\section{Análisis}

En esta etapa se ejecutará todo lo referente al levantamiento de la información de los procesos involucrados, identificando las brechas en el sistema de los procesos actuales y el proceso deseado. Adicionalmente, se inicia el proceso de manejo de cambio tanto para el personal de la organización y los procesos del sistema actual. Se selecciona el equipo de trabajo de acuerdo a las áreas de los procesos del cual se recopiló la información inicial. Se le asignan tareas en cada uno de ellos. Dentro de las definiciones de equipo también se selecciona la metodología de trabajo y la normativa de comunicación entre todos los departamentos interesados del proyecto.

En esta etapa también se eligen por costo-beneficio los contratistas de los sistemas informáticos que se necesiten para la implementación del programa. Se completan los documentos del caso de negocio, el plan del proyecto, y el documento que oficializa el inicio del proyecto firmado por el patrocinador o dueño del proyecto, en donde quedara muy claro el alcance del proyecto, costos, beneficios, riesgos, etc. En esta etapa se debe tener como resultado todos los planes (con tiempos y recursos) y la firma de contrato con la empresa contratista del servicio seleccionado.

Uno de los puntos de referencia más importantes de esta etapa es la reunión de inicio del proyecto, donde se reúne todo el equipo de trabajo y las partes interesadas, para hacer la presentación formal del inicio del proyecto, la presentación del equipo y sus funciones, el plan de actividades del proyecto con sus respectivos responsables y tiempos de cumplimiento, la frecuencia de las reuniones de seguimiento, la medición de los indicadores de desempeño y las fechas de las asignaciones entregables más importantes del proyecto incluido la puesta en marcha operativa del sistema. 
REVISTA FAECO SAPIENS

ISSN L 2644-3821

Acceso Abierto. Disponible en:

https://revistas.up.ac.pa/index.php/faeco sapiens
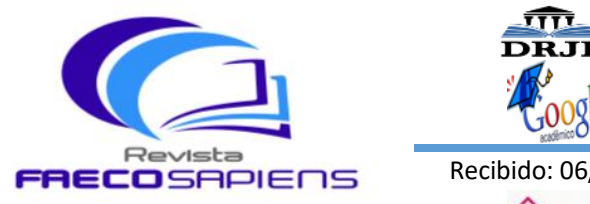

Volumen 4 - Número 1

enero-junio 2021

Recibido: 06/10/20 Aceptado: 19/11/20 pp. 59-73

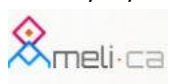

Indexada

Posteriormente a la reunión de inicio del proyecto, se planifican reuniones semanales de seguimientos, en algunos casos y dependiendo del tiempo de implementación, las reuniones podrían ser dos o tres veces por semana, y se utilizan los tableros de indicadores de los registros de acciones, que permite ver las asistencias del equipo de trabajo y las partes interesadas a cada una de ellas, y las tareas realizadas con sus respectivos responsables, fechas de cumplimento y los indicadores de rendimiento claves del proyecto.

\section{Diseño}

En la etapa de diseño se recaba toda la información de los procesos necesarios entre las tablas de SAP y la aplicación web de la empresa contratista del servicio, con esta recopilación de datos se registran los requerimiento de los DEVWR/DEVCR en SAP, para crear la interfaz de la programación de la aplicación de entrada y salida para la creación de los archivos textos conocidos como IDOCs, los cuales le permitirán a SAP producir la información requerida de la factura para ser enviada a la aplicación web del contratista de los servicios, y asimismo de interface de entrada de toda la información que devuelva la aplicación web del consignatario a SAP, como el número único enviado por la agencia tributaria del país, para que sea almacenada en cada registro de factura del programa SAP.

Es indispensable subrayar en esta etapa, que lo más recomendable en ambientes SAP es que la aplicación web del contratista del sistema se respalde con las interfaces creadas para el tema de EDI-SAP (explicado en secciones anteriores de esta investigación), lo cual ahorrara tiempo en la etapa de implementación del sistema. En esta etapa también el contratista del sistema hace toda una revisión con el fin de adaptar su aplicación web, ya sea por requerimientos legales de la agencia tributaria del país, o por configuración específica de SAP dependiendo de temas de seguridad y cumplimiento de la empresa.

\section{Construcción}

En esta etapa del proyecto se comienza a ejecutar los desarrollos levantados en la etapa de diseño, tanto para las interfaces de entrada y salida de SAP, y como las características encajan correctamente con la aplicación web del proveedor. Para los dos casos y como mínimo se debe contar por lo menos con dos ambientes separados de desarrollo: prueba y productivo, en algunas empresas sobre todo en las transnacionales se podría contar hasta con tres ambientes de desarrollo en SAP: regresión, pre-productivo (pruebas), y productivo. Este procedimiento permite que el desarrollo sea primero realizado y probado en un ambiente separado del ambiente productivo, lo que garantiza en el ambiente productivo los tres pilares fundamentales 

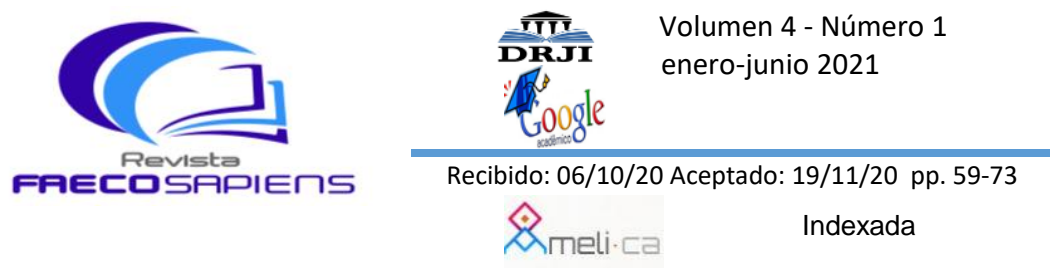

de seguridad informática: confidencialidad, integridad y disponibilidad de los sistemas informáticos. En la práctica real se realizan estas pruebas con la finalidad que el nuevo sistema implementado y los usuarios se adapten a los nuevos procesos del negocio.

Adicionalmente, en esta etapa se implementan todas las pruebas de conectividad desde la aplicación web del contratista del servicio y SAP, empleando los certificados digitales, que han sido obtenidos previamente por la empresa de parte de una entidad certificadora, autorizada por la agencia tributaria del país. Igualmente, la conectividad entre la aplicación web del contratista del servicio, y los servidores y aplicación de la agencia tributaria del país.

\section{Pruebas}

En la etapa de pruebas se recomienda realizarlo en ambientes de pre-productivo de SAP:

a. En primer lugar se prueban todos los desarrollos realizados entre las áreas de tecnología tanto de la empresa como por la del contratista del servicio, a esta etapa se les conoce como pruebas MIT.

b. Inmediatamente después de aprobadas y certificadas las pruebas MIT, se proceda hacer los adiestramientos del sistema con los usuarios finales de la empresa, tanto de los cambios realizados en SAP, como de la nueva herramienta web a utilizar del contratista del servicio.

c. Posteriormente a este entrenamiento se realizan pruebas completas del nuevo sistema, entre todas las partes involucradas, para certificar que todos los procesos implementados funcionan según el requerimiento de los datos recabados inicialmente. A estas etapas de pruebas se les conoce como pruebas MAT.

En ocasión de que las pruebas (MIT o MAT) no sean satisfactorias, y se requiera de nuevas modificaciones al sistema ya sea en las interfaces de SAP o en la aplicación web del contratista del sistema, el ciclo de pruebas nuevamente inicia en el primer punto de esta explicación.

\section{Documentación y entrenamiento}

Una vez certificada por los usuarios finales la etapa de pruebas, todos los cambios en SAP deben ser documentados y entregados a los usuarios finales. De igual manera el contratista del sistema debe entregar un manual de usuario de la aplicación web, la cual también será entregada a los usuarios que de manera directa van a interactuar con el sistema. 
Acceso Abierto. Disponible en:
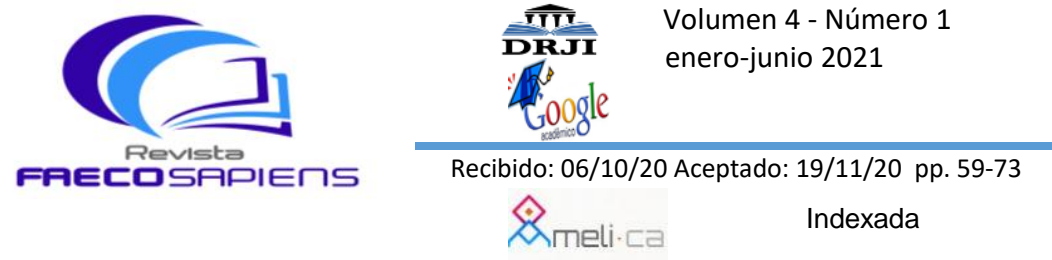

En esta etapa se crea el plan de corte del sistema, que es un detalle de las actividades a realizar previo a la fecha de puesta en marcha del sistema. Por lo general estas actividades incluyen tareas como:

a. Adiestramientos adicionales a los usuarios finales. Esta etapa es crítica debido a que de no entrenar correctamente al personal pueden crear fallas en los registros contables posteriores.

b. Ratificación de los accesos a los sistemas de los usuarios finales. En esta etapa se le asigna el perfil de SAP de acuerdo a sus tareas y responsabilidades.

c. Comprobación de soporte para el día de puesta en marcha del sistema tanto de SAP como para la aplicación web del contratista del servicio.

d. Convalidación de la aprobación a la etapa del ambiente productivo: SAP y aplicación del contratista del servicio.

e. Reunión avance/no avance con el dueño del proyecto y todas las partes interesadas del proyecto, en donde se acepta el proyecto y el día de la puesta en marcha del sistema.

f. En esta etapa se comparte el estado y situación del trabajo de forma diaria, sobre todo cuando ya falte solo una semana para la puesta en marcha del sistema.

\section{Puesta en marcha y cierre del proyecto}

Cuando se habla de una salida en vivo es cuando se pone en marcha el sistema. El día de la salida en vivo del proyecto, previamente se ha tomado la decisión de común acuerdo con los usuarios finales para dar inicio a la etapa del sistema de forma controlada, que no es más que el uso de las aplicaciones SAP y la del contratista de los servicios web, pero utilizando pocas facturas o clientes, para asegurar que todos los cambios en SAP están ocurriendo tal como fueron validados en las pruebas anteriores. En esta puesta en marcha del sistema controlado se permite también en caso de alguna eventualidad hacer las correcciones necesarias sin que se vea afectada considerable la información del cliente. El día de la puesta en marcha del nuevo sistema es preciso la participación de todos los miembros del equipo y del personal técnico del contratista del servicio, podrían ser opcionales el dueño del proyecto y las partes interesadas, pero por lo general también participan sobre todo si son proyectos de alta prioridad para los directivos de la empresa.

Desde la aplicación web del contratista del sistema se realizará la impresión física de la factura. Posteriormente que se haya comprobado de que todos los cambios en ambientes productivos funcionen de acuerdo con lo solicitado y esperado, la aplicación pasa por un período de "hipercuidado", con la expectativa que su 
REVISTA FAECO SAPIENS

ISSN L 2644-3821

Acceso Abierto. Disponible en:

https://revistas.up.ac.pa/index.php/faeco sapiens
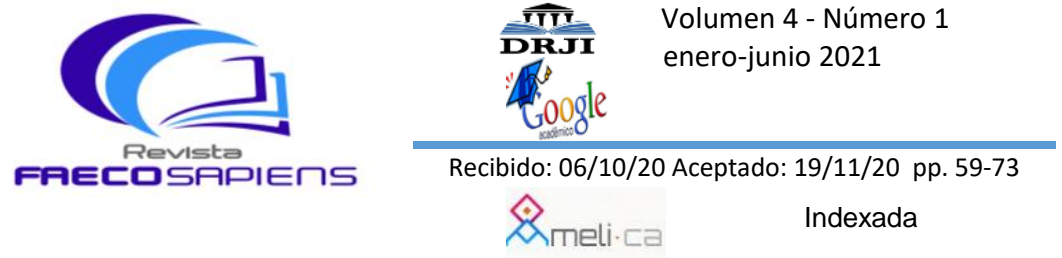

rendimiento sea el esperado, y en cualquier situación adversa sea reportado a todos los equipos para que se le brinde la asistencia técnica en el menor tiempo posible.

Habitualmente en estos periodos de "hipercuidado" es de 14 a 30 días, con el fin de mejorar el rendimiento de la información administrada. En la etapa de "hipercuidado" se pueden tener reuniones semanales, para evaluar el rendimiento de los sistemas informáticos implementados en ambiente productivo. Luego de pasado el período de "hipercuidado", el dueño del proyecto debe llenar la encuesta de satisfacción de la implementación del proyecto, con el fin de recabar información del conocimiento adquirido sobre los procesos implementados, a través de reflexiones críticas sobre los factores que pueden haber afectado positiva o negativamente la implementación del nuevo sistema.

\section{CONCLUSIONES}

Los gobiernos se están beneficiando de las implementaciones de la facturación electrónica a nivel mundial. Debido a que maximiza la lucha contra el fraude fiscal. Después de analizar y revisar toda la documentación obtenida para esta investigación, no hay duda de que el flujo correcto de las fases en una metodología tradicional (cascada), pueden ser útiles para lograr confeccionar los análisis, planes, actividades y control requeridos, para tener una entrega de producto con alta calidad. También se pudo comprobar que es posible reutilizar los IDOCs de EDISAP para la generación de facturas electrónicas.

El sistema SAP R/3 opera utilizando el principio cliente/servidor aplicado a varios niveles. Para cumplir con las normativas de la facturación electrónica en los diferentes países es necesario no utilizar soluciones aisladas, sino contar con una solución que sea modular y que utilice fundamentalmente por medio del software, de forma que los modos de interacción entre los diversos clientes y servidores puedan ser controlados. La factura electrónica, es un tipo de factura que se diferencia de la factura en papel por la forma de gestión informática y el envío mediante un sistema de comunicaciones que conjuntamente permiten garantizar la autenticidad y la integridad del documento electrónico. Siendo de gran beneficio para los consumidores la generación de facturas electrónicas por las razones expuestas en esta investigación, y que aunque no se cuente con un papel físico, también se puede obtener una representación impresa desde los sitios web. El modelo de facturación que se adopte utilizando SAP/R3 en cada país se debe de cumplir con las reglamentaciones definidas por la normatividad legal de cada país. 


\section{REFERENCIAS BIBLIOGRÁFICAS}

Álvarez, C. (2015). Adaptación de las Metodologías Tradicionales Cascada y Espiral para la Inclusión de Evaluación Inicial de Usabilidad en el Desarrollo de Productos de Software en México.

Barraza de Wong, A. (2018). El Capital Financiero, Consideraciones frente a la Factura Electrónica en Panamá, Recuperado de: https://elcapitalfinanciero.com/consideraciones-frente-a-la-facturaelectronica-en-panama/

Blog de Solorio (2013). M. Metodología en Cascada. Recuperado de: http://metodologiaencascada.blogspot.com/

Debitoor (2019). Factura Electrónica. Recuperado de: https://debitoor.es/glosario/definicion-facturaelectronica.

Digital Guide IONOS (2019). El modelo en cascada: desarrollo secuencial de software.

Digital Guide IONOS. Recuperado de: https://www.ionos.es/digitalguide/paginas-web/desarrolloweb/el-modelo-en-cascada/.

ENCAT (2014). Evolución Factura Electrónica en América Latina.

Escudero, I. (2017). Intercambio de información EDI. Qué es y su integración con SAP. Recuperado de: https://www.sothis.tech/intercambio-informacion-edi-integracion-sap/.

Go Anywhere (2019). ¿Qué es EDIFACT? Go Anywhere. Recuperado de: https://www.goanywhere.com/es/blog/que-es-edifact

Guru99 (2019). ALE, EDI e IDOCs Introducción y diferencia: Tutorial de SAP, Recuperado de: https://www.guru99.com/what-is-edi-ale-and-idoc.html.

Intercambio Electrónico de Datos, EDI. (2020). Seres Grupo Dacaposte. Recuperado de: https://co.groupseres.com/edi/que-necesita.

Intercambio de la Información EDI (2019). Sothis Tech. Recuperado de: https://www.sothis.tech/intercambio-informacion-edi-integracion-sap/.

Luque, R. (2019). Guía práctica para la implementación de factura electrónica.

Mejías, A. (2013). Interoperabilidad de módulos del sistema R/3 de SAP. Ministerio de Economía y Finanzas. (2019). Dirección General de Ingresos de Panamá: Recuperado de https://dgi.mef.gob.pa/facturaelectronica/index.html.

Muñoz, M. (2014). El Blog de las PYMES. Recuperado de: http://blog.deudae.com/8-ventajas-de-lafactura-electronica/.

NexTech Education Center (2017). ¿Qué es SAP y para qué sirve? Recuperado de: https://nextech.pe/que-es-sap-y-para-que-sirve-sap/. 


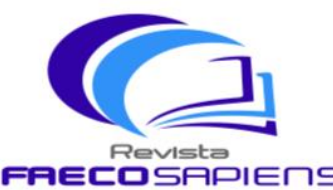

คमEСロSคDIEחS

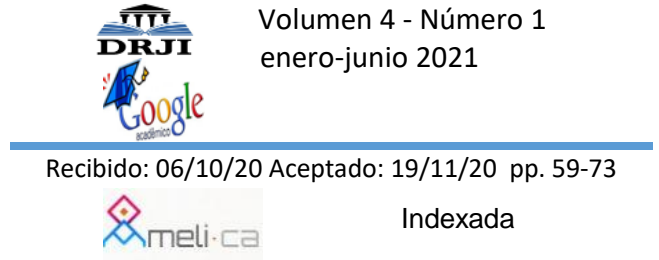

Novis (2018). Una metodología ágil para las mejoras de SAP. Recuperado de: https://www.novis.cl/noticias-novis/servicios-sap-2/una-metodologia-agil-para-las-mejoras-sap/.

TIC Portal, (2019). ¿Qué es un sistema ERP y para qué sirve? Recuperado de: https://www.ticportal.es/temas/enterprise-resource-planning/que-es-sistema-erp.

Universidad Politécnica de Valencia. ¿Qué es un Certificado Digital? (2012). Universidad Politécnica de Valencia. Recuperado de: https://www.upv.es/contenidos/CD/info/711545normalc.html.

Valdés, Y. (2019). El Panamá América. Factura electrónica, la nueva forma de comprar. Recuperado de: $\quad$ https://www.panamaamerica.com.pa/economia/factura-electronica-la-nueva-forma-decomprar-11 $\overline{28888}$ 